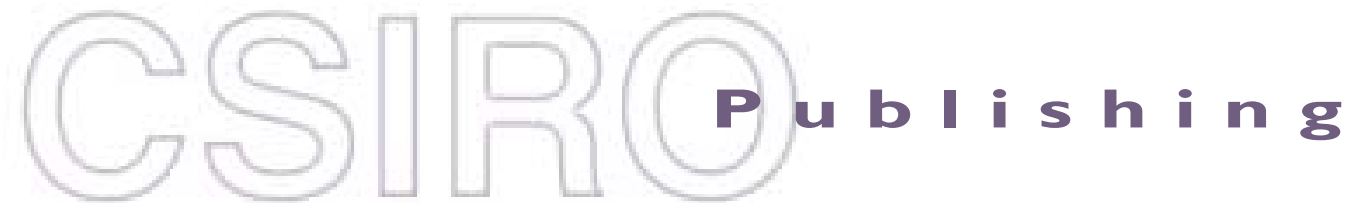

\section{Publications of the Astronomical Society of Australia}

Volume 19, 2002

(C) Astronomical Society of Australia 2002

An international journal of astronomy and astrophysics

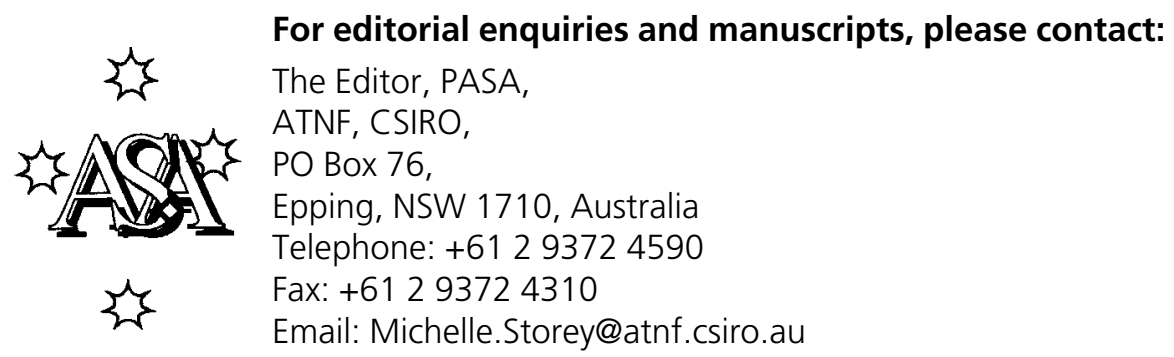

For general enquiries and subscriptions, please contact: CSIRO Publishing PO Box 1139 (150 Oxford St)

Collingwood, Vic. 3066, Australia

Telephone: +6139662 7666

Fax: +61 396627555

Email: publishing.pasa@csiro.au

C S I RO

PUBLISHING Published by CSIRO Publishing

for the Astronomical Society of Australia

www.publish.csiro.au/journals/pasa 


\title{
Radiation Fields in Blazars - a Possible Extension of the Small Scale Symbiosis (Disk/Jet) into a Large Scale (Dust/Dust) Symbiosis
}

\author{
Alina-C. Donea and Raymond J. Protheroe \\ Department of Physics and Mathematical Physics, \\ University of Adelaide, SA 5001, Australia \\ adonea@physics.adelaide.edu.au \\ Received 2001 August 4, accepted 2001 November 7
}

\begin{abstract}
In blazar models both protons and electrons may be efficiently accelerated in jets and produce $\gamma$-rays. Here we discuss the interactions of these $\gamma$-rays with different radiation fields. The external radiation fields within a few parsecs from the black hole involved in such interactions could be the direct radiation from the accretion disk coupled with the jet, the infrared radiation from a dusty torus, and the emission line radiation from the broad line region surrounding the accretion disk. The optical thickness for absorption of $\gamma$-ray photons in the external radiation fields is analysed for blazars and quasars.

Based on the unification theory of active galactic nuclei we briefly review the evidence for the existence of small scale dust tori in blazars/FR I. We propose that the existing jet-accretion disk symbiosis extrapolates to a large scale symbiosis between other important dusty constituents of the blazar/FR I family.
\end{abstract}

Keywords: proton blazar model — accretion disks — dust torus — broad line region — jet

\section{Introduction}

We address the problem of where the $\gamma$-ray emission region is located along the jet in active galactic nuclei (AGN) and especially in blazars. This is an important issue since the optical thickness for $\mathrm{GeV}$ and $\mathrm{TeV}$ photons interacting with the external radiation fields of AGN can affect the $\gamma$-ray spectral energy distribution.

Despite the large number of blazars detected by EGRET, only four of them (Mrk 501, Mrk 421, 1ES 2344514, and PKS 2155-304) show TeV emission. No quasar has yet been observed at $\mathrm{TeV}$ energies and this suggests that, since they are located usually at high redshifts, the IR background radiation could have substantially absorbed the $\mathrm{TeV}$ photons (Stecker \& de Jager 1998). An alternative explanation could be that $\mathrm{TeV}$ photons from quasars were already absorbed in the inner parsec region. For example, the infrared emission produced by tori could inhibit the escape of $\mathrm{TeV} \gamma$-rays due to photon-photon pair-production (Protheroe \& Biermann 1997).

Since unification schemes propose that FR I and BL Lacs are related, FR I sources being the misoriented counterparts of BL Lacs (Urry \& Padovani 1995), we have searched the literature for information about dust structures associated with FR I. Since blazars, in fact, represent a large family of objects (optically violent variables (OVV), high-polarisation quasars, BL Lac objects), an understanding of the physics of one of these objects may lead to a clarification of the properties of all objects of the same class.

There are two issues which should be considered in connection with dust tori in blazars. Firstly, since there is no direct evidence of any thermal emission from tori we should consider what the observations tell us, indirectly, about the existence of dust at the centres of host galaxies.
Secondly, what is the kind of torus geometry that would fit the observations made at different wavelengths best. A possible link between the torus and the broad line region (BLR) is outlined in our discussion.

\section{Unification}

The key point in our work is that a unification theory has already been demonstrated for several classes of AGN. Relativistic outflows are very nicely described by a model of the small scale symbiosis between the accretion disk and jet (Falcke, Gopal-Krishna, \& Biermann 1995, and references therein; Donea \& Biermann 1996) for the case where the base of the jet has radius $R_{\text {jet }} \leq 10 R_{g}=$ $10^{-4} M_{8} \mathrm{pc}\left(1 \mathrm{pc}=3 \cdot 10^{16} \mathrm{~m}\right)$, where $M_{8}=M / 10^{8} M_{\odot}$. This model seems to work well for different types of AGN. We adopt a classical picture of the inner parsec-scale region of an AGN with a Kerr black hole, accretion disk, jet, torus, and BLR (five components). In Seyfert galaxies and quasars the torus and the BLR are active components, as can be inferred from the large forest of observed emission lines. In the case of blazars, any emission from the torus would be dwarfed by the non-thermal radiation from the jet, making impossible the direct detection of the inner dust structure. However, in order to explain the OVV $\gamma$-ray spectra, Blazejowski et al. (2000) required, in addition to the self synchrotron Compton model, an external IR photon field scattered by relativistic jet electrons in order to account for the observed EGRET fluxes.

We ask ourselves: 'Why should blazars not have the same five natural parsec-scale components which seem to fit a large variety of objects?'

Despite the fact that the majority of BL Lacs do not show any type of emission lines, Corbett et al. (2000) confirmed the presence of a weak broad $H_{\alpha}$ emission line 
in BL Lacertae. Even Mrk 421 seems to have a BLR with a very low luminosity $L_{\mathrm{BLR}}=1.5 \cdot 10^{40} \mathrm{erg} \mathrm{s}^{-1}=$ $1.5 \cdot 10^{33} \mathrm{~W}$ (Morganti, Ulrich, \& Tadhunter 1992). In addition, Chiaberge, Capetti, \& Celloti (1999) have concluded that BLRs and obscuring tori are closely linked, and both are present only in association with accretion.

When there is no detection of direct optical emission, the interpretation of spectropolarimetric data on FR I objects with strong evidence for infrared obscuration (Antonucci 2001, and references therein) could suggest some properties of BLRs hidden by thick tori. The observed polarised broad lines would be the result of scattering into the line of sight by free electrons in zones whose geometry and orientation is dependent on the torus' inner geometry. Hence, the detection of free electron scattering regions should be a good diagnostic of the geometry of the inner region of the torus. Falcke et al. (1995) proposed that the opening angle of the torus might play a critical role for the FR I and FR II dichotomy; a closed torus covering a large fraction of $4 \pi$ steradians as seen from the black hole would obscure the internal activity of FR I objects. The obscuration of BLRs by tori is an impediment to finding the right BLR-disk system luminosity.

\section{Large Scale and Small Scale Tori: Dust Everywhere}

We are interested in observational evidence for the existence of tori in blazars/BL Lacs/FR I. We have looked into the literature, and found that several FR I show incontestable proof of having torus dust structures: Centaurus A (Alexander et al. 1999), 3C270 (Barth, Filippenko, \& Moran 1999), and 3C218 (Sambruna et al. 2000; Antonucci 2001). At kiloparsec scales the contribution from starbursts dominates the infrared emission, but as one goes deeper into the nucleus of the galaxy, de Koff et al. (2000) found that well-organised dust structures tend to develop in 3CR objects. This is a very important result, as they emphasised that dust in FR I host galaxies is generally situated in sharply defined small scale disks $(\leq 2.5 \mathrm{pc})$.

The detection of large scale dusty features (sometimes shaped as bars or dust lanes) suggests that there could be an association between the small scale infrared torus, such as those most Seyfert galaxies display, and a large scale torus sometimes identified with the external dust structure of the galaxy. The structure of the nuclear torus would then depend on the large scale dust distribution - it appears that well-organised kpc structures extend inward, towards smaller scales. We define this as a symbiosis between the large and small scale dusty features.

HST observations have shown the presence of dust in a large fraction of radio galaxies. The dust is often seen to be clumpy in FR II, but not clumpy in FR I (de Koff et al. 2000), and this could indicate that the flow of matter towards the nucleus is rather steady in FR I, allowing for the formation of distinct torus features. We suggest that the kpc-scale dust could be a reservoir of dust which will be accreted towards the central nucleus, and that a highly variable flow would not facilitate the formation of welldefined dusty structures. This conjecture would also fit with the Seyfert galaxy geometry, where there is direct evidence for the existence of stable tori.

\section{Radiation Fields in AGN and the $\gamma-\gamma$ Opacity}

The radiation fields dominant at parsec scales in AGN are accretion disk radiation (anisotropic), line emission from BLR clouds (also anisotropic), and infrared emission from a heated dusty torus. The small scale symbiosis is reflected mostly in a modified distribution of photons from the inner region of the accretion disk in which the jet is anchored (Donea \& Biermann 1996). This is important for calculating $\gamma$-ray spectra when disk activity is involved, especially in the flaring states. The flaring state of a blazar could be related to an increase of the energy accumulated in the inner disk and subsequently expelled into the jet.

The disk luminosity $L_{\text {DISK }}$ and the power of the jet $Q_{\text {JET }}$ are then controlled by conditions at the base of the jet. The luminosity of a disk in symbiosis with a jet is $L_{\mathrm{DISKJ}}=L_{\mathrm{DISK}}-Q_{\mathrm{JET}}$, and $L_{\mathrm{DISKJ}}=\xi_{\mathrm{SYMB}} L_{\mathrm{DISK}}$, where $\xi_{\mathrm{SYMB}} \leq 1$ is the parameter for the jet-disk connection.

For blazars the central luminosity is $L_{\mathrm{UV}} \approx 10^{44}-10^{46}$ $\mathrm{erg} \mathrm{s}^{-1}$. We take a value of $L_{\mathrm{UV}}=10^{46} \mathrm{erg} \mathrm{s}^{-1}$ for our examples discussed below. The energy density of disk photons at distance $r$ is $U_{\text {DISKJ }}=L_{\text {DISKJ }} / 4 \pi c r^{2}$.

We postulate that the BLR and torus are linked elements, and we shall now define the geometry of this system. The inner radius of the torus is determined by the sublimation radius of the dust in the torus, $R_{\text {in,torus }} \sim T_{s, 1500}^{-2.8} L_{46}^{1 / 2}$ pc (Danese et al. 1998), where $L_{\mathrm{UV}}=10^{46} L_{46} \mathrm{erg} \mathrm{s}^{-1}$, and the dust is heated to a temperature $T=10^{3} \mathrm{~K}$. Here, we consider a small scale torus having height $H=3.3 \mathrm{pc}$, inner radius $A=1 \mathrm{pc}$, and outer radius $B=2 \mathrm{pc}(A / H=0.3$, Pier \& Krolik 1992).

The BLR is represented by a spherical shell of small clouds with number density varying as $N_{\text {cloud }} \sim$ $\left(r / r_{\text {in, BLR }}\right)^{-\alpha}$ and cloud radii varying as $R_{\text {cloud }} \sim$ $\left(r / r_{\text {in,BLR }}\right)^{-\beta}(\alpha=1.5$ and $\beta=-0.6$, Kaspi \& Netzer 1999 ), extending between radii $r_{\mathrm{in}, \mathrm{BLR}}=0.01 \mathrm{pc}$ and $r_{\text {out,BLR }}=0.4 \mathrm{pc}$ (Hartman et al. 2001). We consider that the BLR is optically thin. The optical depth is assumed to be $\tau=0.01$ giving a total BLR luminosity of $L_{\mathrm{BLR}}=10^{44} \mathrm{erg} \mathrm{s}^{-1}$. Accretion disk photons excite the BLR material, and emission lines such as $H_{\alpha}, H_{\beta}, O I I I$, $N I I$, etc. are produced. We simplify our calculation by assuming that the entire BLR luminosity is emitted in the $H_{\alpha}(6563 \AA)$ line. The full angular dependence of the BLR $H_{\alpha}$ intensity at different positions $r$ along the jet is taken into account. At $r>r_{\text {out,BLR }}$ the BLR photon energy density is $u_{\mathrm{BLR}} \approx L_{\mathrm{BLR}} / 4 \pi c r^{2}$.

At different positions along the jet, the energy density of one of the three radiation fields dominates. We find that for the present assumptions, for $r>0.6 \mathrm{pc}$ the contribution from the UV disk photons decreases significantly, while 


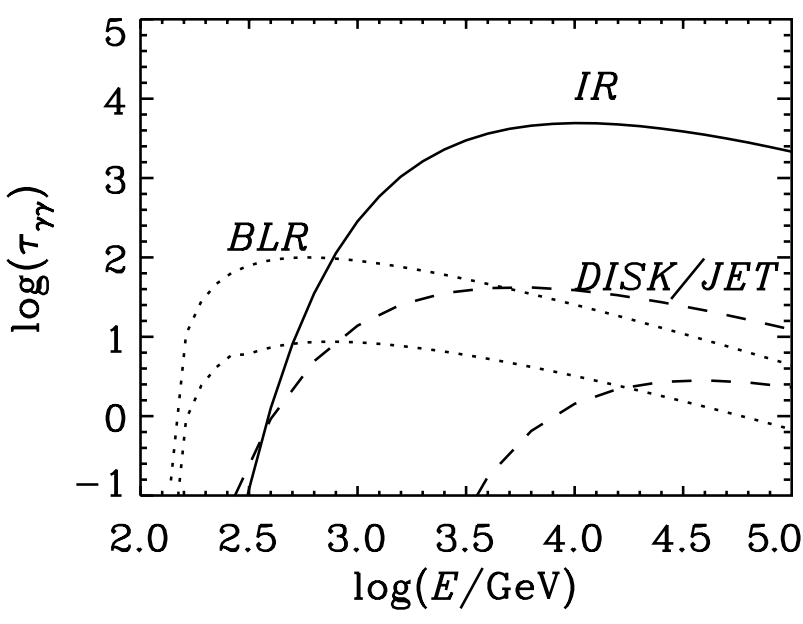

Figure 1 Optical depths for BLR photons interacting with $\gamma$-rays produced at different locations in the jet: $r=0.01 \mathrm{pc}$ (top dotted curve), $r=0.1 \mathrm{pc}$ (bottom dotted curve). The curve labelled 'IR' shows the contribution from IR photons interacting with jet photons emitted at $0.1 \mathrm{pc}$. The dashed curves labelled 'DISK/JET' correspond to interactions with the accretion disk radiation for $r=0.01 \mathrm{pc}$ (top dashed curve) and $r=0.1 \mathrm{pc}$ (bottom dashed curve) with $L_{\mathrm{UV}}=10^{46} \mathrm{erg} \mathrm{s}^{-1}$ and $L_{\mathrm{BLR}} \approx 10^{44} \mathrm{erg} \mathrm{s}^{-1}$.

the energy density of isotropic IR radiation $U_{\text {IR }}$ remains constant over a scale equal to the height of the torus. For $r<0.6 \mathrm{pc}, U_{\mathrm{DISKJ}}>U_{\mathrm{IR}}$. The energy density of BLR photons peaks where the ionising contribution from the disk photons dominates, i.e. $r \approx r_{\text {in, BLR }}$.

The radiation fields present within the parsec scale of AGN may create a problem for the escape of high energy $\gamma$-rays. We calculate the optical depth for photon-photon pair production in the radiation fields of the disk/jet, BLR, and torus. The optical depth for escaping $\gamma$-rays at various locations along the jet axis is shown in Figure 1. The contribution from each radiation field is labelled 'DISK/JET', 'BLR', and 'IR', respectively.

The BLR can be either completely hidden by a closed torus, or partially covered by an open torus. This makes an exact estimation of $L_{\mathrm{BLR}}$ difficult. For quasars, the BLR region would absorb $10^{2}-10^{3} \mathrm{GeV}$ photons if they are produced within the very inner region of the BLR where the energy density of BLR photons has a maximum value. Low values of $L_{\mathrm{BLR}}$ could describe objects with weak broad line emission such as blazars. Also, since $L_{\text {disk }}$ could be less than $10^{46} \mathrm{erg} \mathrm{s}^{-1}$ in blazars, we expect that $L_{\mathrm{BLR}}<10^{44-45} \mathrm{erg} \mathrm{s}^{-1}$. A low value of $L_{\text {BLR }}$ would make the optical depth $\tau_{\gamma \gamma \text {,BLR }}$ insignificant unless the local distribution of BLR clouds depends strongly on radius.

As noted previously by Protheroe \& Biermann (1997), we can see from the curve labelled 'IR' that for photon energies above about several hundreds of $\mathrm{GeV}$ the opacity is large enough that no radiation can emerge if the source of $\gamma$-rays is near the centre of the torus. If $\eta_{\mathrm{IR}}$ is the efficiency of reprocessing by the torus of the disk photons, then $u_{\mathrm{IR}} \approx \eta_{\mathrm{IR}} a T^{4} \mathrm{erg} / \mathrm{cm}^{3}$, where $a$ is the radiation constant.
Then the optical depth due to the torus would be reduced by factor $\eta_{\mathrm{IR}}$. Even for extremely low values, e.g. $\eta_{\mathrm{IR}}=10^{-4}$, absorption of the torus radiation can be significant. We note, however, that Blazejowski et al. (2000) require $\eta_{\mathrm{IR}} \approx 0.3$ to fit $\gamma$-ray spectra of OVV with an external inverse Compton model. We also found that an open torus geometry would only slightly modify the optical depth IR curve in Figure 1.

Our results show that the attenuation of $\gamma$-rays emitted from the jet by interaction with photons from the BLR, as well as photons from the IR torus and from the disk (in symbiosis with the jet) is important for imposing limits on the position of the $\gamma$-ray emitting blob.

Since the $\gamma-\gamma$ opacity for $10^{2}-10^{3} \mathrm{GeV}$ photons is large when the emitting region is near the inner radius of the BLR we conclude that the blob should be located at $r>0.02 \mathrm{pc}$ (for $L_{\mathrm{BLR}}=10^{44} \mathrm{erg} \mathrm{s}^{-1}$ ). As one goes farther out along the jet, GeV photons escape. The situation is different for $\mathrm{TeV}$ photons which could emerge from the blazar only if the emitting region is located at parsec distances from the black hole, far above the torus. This is in accordance with the results of Valtaoja (2001), who concluded that the temporal connection between the radio and $\gamma$-ray variations suggests that the $\gamma$-rays appear to originate far away from the radio core.

We conclude that the origin of the $100-1000 \mathrm{GeV} \gamma$-ray photons must be above the inner part of the BLR region for any AGN. Blazars usually have low central luminosities and this translates into a low $L_{\mathrm{BLR}}$. The BLR region can still absorb $\gamma$-rays above $100 \mathrm{GeV}$ if $L_{\mathrm{BLR}} \geq 10^{42} \mathrm{erg} \mathrm{s}^{-1}$ and the emitting region is very close to its inner radius, while $\mathrm{TeV} \gamma$-ray photons must be produced far above the infrared torus. However, Mrk 421 seems to have an extremely low $L_{\mathrm{BLR}} \approx 1.5 \cdot 10^{40} \mathrm{erg} \mathrm{s}^{-1}$ (Morganti et al. 1992) and this suggests that the BLR could be everywhere transparent to photon-photon absorption. This gives us an insight into the sites of energetic particle acceleration along AGN jets. At parsec distances above the accretion disk all external radiation fields apart from the infrared radiation cease to be important. In this case the intrinsic radiation of the emission regions in the jet becomes dominant.

The radiation fields discussed in this paper may also provide important target photons for interactions of accelerated electrons or protons, and this will be discussed in a future paper.

\section{References}

Alexander, D. M., et al. 1999, MNRAS, 310, 78

Antonucci, R. 2001, astro-ph/0103048

Barth, A. J., Filippenko, A. V., \& Moran, E. C. 1999, ApJ, 525, 673

Blazejowski, M., Sikora, M., Moderski, R., \& Madejski, G. M. 2000, ApJ, 545, 107

Chiaberge, M., Capetti, A., \& Celloti, A. 1999, A\&A, 349, 77

Corbett, E. A., Robinson, A., Axon, D. J., \& Hough, J. H. 2000, MNRAS, 311, 485

Danese, L., Silva, L., Granato, G. L., \& Franceschini, A. 1998, ApJ, 509, 103

de Koff, S., et al. 2000, ApJS, 129, 33 
Donea, A.-C., \& Biermann, P. L. 1996, A\&A, 316, 43

Falcke, H., Gopal-Krishna, \& Biermann, P. L. 1995, A\&A 298, 395

Kaspi, S., \& Netzer, H. 1999, ApJ, 524, 71

Morganti, R., Ulrich, M.-H., \& Tadhunter, C. N. 1992, MNRAS, 254,546

Hartman, R. C., et al. 2001, ApJ, 553, 683
Pier, E. A., \& Krolik, J. H. 1992, ApJ, 401, 99

Protheroe, R. J., \& Biermann, P. L. 1997, Astropart. Phys., 6, 293 Sambruna, R. M., Chartas, G., Eracleous, M., Mushotzky, R. F., \& Nousek, A. J. 2000, ApJ, 532, L91

Stecker, F. W., \& de Jager, O. C. 1998, A\&A, 334, L85

Urry, C. M., \& Padovani, P. 1995, PASP, 107, 803

Valtaoja, E. 2001, PASA, 19, in press 